\title{
Technology-enhanced feedback
}

\author{
Ameena Payne
}

Potential competing interests: The author(s) declared that no potential competing interests exist.

At its simplest this involves annotating documents with written comments.

May also include audio-visual feedback: A feedback modality that is captured via a digital recording of a computer screen (e.g. screen-cast or screen-capture) and provided to students by higher education tutors. 